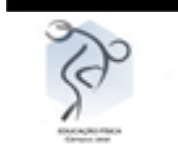

\title{
Perfil e prevalência de lesões osteomusculares de praticantes de exercício físico supervisionado em academias
}

\author{
Daniel Cortes Beretta ${ }^{1}$ \\ Camila Fernandes da Costa ${ }^{2}$ \\ Sabrina Tofolli Leite ${ }^{3}$ \\ Chaysther de Andrade Lopes ${ }^{4}$ \\ Rênica Alves de Morais Rocha ${ }^{5}$ \\ David Michel de Oliveira ${ }^{6}$
}

Resumo: a prática regular de exercícios físicos é importante estratégia para melhora da aptidão física e melhora da saúde, entretanto quando não executados com métodos adequados resultam em lesões osteoarticulares. O objetivo deste estudo foi Identificar o perfil de praticantes de exercício físico supervisionado e verificar a prevalência de lesões osteomusculares. Este estudo descritivo foi realizado em academias de ginástica da cidade de Jataí-GO. A análise foi realizada por meio da aplicação de um questionário padrão estruturado, que abordou sobre idade, sexo, atributos físicos, hábitos de atividades físicas e percepção de lesões. Foi aplicado um total de 200 questionários. Após tabulados os dados foram submetidos à análise estatística pelo programa IBM SPSS Statistics versão 2.0 e realizado o teste do Qui quadrado com o recurso da análise de V de Cramer para avaliar as possíveis relações entre as variáveis. Dos 200 entrevistados apenas 33 responderam terem tido tendinite. A dor foi o principal sintoma relatado pelos entrevistados com tendinite. Os exercícios realizados de maneira adequada e bem orientados refletiram-se na ausência de dor e lesões. Porém, quando praticados de maneira errônea e com excesso de sobrecarga resultaram em lesões como a tendinite. A partir disso, enfatiza-se a importância do conhecimento e detecção da doença por parte dos praticantes de musculação para que possam procurar ajuda médica prévia, realizar o devido tratamento e evitar o afastamento momentâneo ou definitivo das atividades físicas.

Palavras -chave: Musculação. Tendinopatia. Exercícios físicos.

\section{Profile and prevalence of osteomuscular injuries of supervised physical exercise practitioners in academies}

Abstract: regular exercise is an important strategy for improving physical fitness and health, however when not performed with appropriate methods, they result in osteoarticular injuries. The aim of this

\footnotetext{
${ }^{1}$ Docente do curso de Biomedicina da Universidade Federal de Jataí e Chefe da UAECISAU (UFJ) E-mail: berettadc@hotmail.com

${ }^{2}$ Bacharel em Biomedicina pela Universidade Federal de Goiás/REJ E-mail: camila_alves_fernandes@hotmail.com

${ }^{3}$ Docente do curso de Educação Física da Universidade Federal de Jataí e Sub-Chefe da UAECISAU (UFJ). Email: sabrina.toffoli@gmail.com

${ }^{4}$ Docente do curso de Educação Física da Universidade Federal de Jataí (UFJ). E-mail:Chaystherlopes@hotmail.com

5 Mestra do Programa de Ciências aplicadas a Saúde da Universidade Federal de Goiás (UFG/REJ)

E-mail: renicamorais@hotmail.com

${ }^{6}$ Docente do curso de Educação Física da Universidade Federal de Jataí (UFJ).

E-mail: profdoliveira@gmail.com
} 


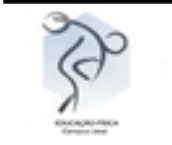

study was to identify the profile of supervised physical exercise practitioners and to verify the prevalence of osteomuscular injuries. This descriptive study was conducted in the gyms of the city of Jataí-GO. The analysis was performed by applying a structured standard questionnaire, which addressed on age, gender, physical attributes, physical activity habits and perception of injury. A total of 200 questionnaires were applied. After tabulation, the data were subjected to statistical analysis using the IBM SPSS Statistics version 2.0 software and chi-square test was performed using cramer's $\mathrm{V}$ analysis to evaluate possible relationships between variables. Of the 200 respondents only 33 said having had tendonitis. Pain was the main symptom reported by respondents with tendonitis. The exercises performed properly and well oriented reflected in the absence of pain and injuries. However, when practiced wrongly and with excessive overload resulted in injuries such as tendonitis. From this, it emphasizes the importance of knowledge and detection of diseases by bodybuilders so that they seek prior medical help, perform the treatment and avoid the momentary or definitive departure from the physical activities.

Keywords: Bodybuilding. Tendinopathy. Physical exercises.

\section{INTRODUÇÃO}

Nos últimos anos o número de adeptos a prática de atividades físicas supervisionadas em academias aumentou vertiginosamente devido ao reconhecimento cientifico e midiático de seus benefícios biopsicossociais e ganhos paralelos à saúde (SABA, 2004). Os praticantes procuram além da melhoria na qualidade de vida e promoção da saúde, uma autoimagem mais positiva (LIZ et.al., 2010). O desejo pelo corpo perfeito entre homens e mulheres, para se adequarem à modelos pré estabelecido pela sociedade, reflete diretamente no investimento de tempo e recursos financeiros em serviços oferecidos pelas academias de ginástica (IRIART et. al., 2009). Atualmente, as academias são reconhecidas pela diversidade de modalidades oferecidas com o intuito de induzirem a pratica de atividades físicas constantes. Diante do modismo imposto pela sociedade, percebe-se que os treinos com grande intensidade, duração e carga, passaram a ser mais utilizados como meta para o ganho de massa muscular. (TAHARA et.al., 2003).

Os Sistemas e métodos de treinamento físico são fundamentais para o alcance de um corpo saudável, porém, quando realizados de forma incorreta ou sem orientação profissional, resultam em lesões osteoarticulares em diversas regiões do corpo. Essas lesões estão diretamente relacionadas com o método ou sistema de treinamento e devido a execução 


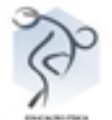

do exercício, sobrecarga progressiva de peso, séries extenuantes e postura inadequada (GOMES, 2013).

Os tendões são constituídos basicamente por fibras colágenas, o que lhes conferem flexibilidade e facilita a locomoção dos indivíduos. Isso torna possível os movimentos que requerem força e agilidade, melhorando a qualidade do exercício proposto e atingindo a amplitude máxima do movimento. (CORTES et.al., 2002). As lesões tendíneas são as mais comuns no meio esportivo, e são reflexo de situações traumáticas ou do uso das estruturas anatômicas de modo excessivo e repetitivo (PRADO, 2013). A inflamação dos tendões é caracterizada pela tendinite. Esse quadro clínico é evidenciado por dor no local lesionado, calor, aumento do volume tecidual e dificuldade em realizar movimentos que requerem força. As lesões tendíneas possuem caráter progressivo que podem culminar em ruptura do tendão e consequente suspensão da atividade física. O diagnóstico precoce e precauções em relação à essa enfermidade podem evitar prejuízos maiores aos praticantes de academia. Por isso, é preciso elaborar formas de prevenir e de identificar a incidência de lesões que ocorram da execução errônea de exercícios físicos (LAURINO, 2008). O presente estudo surgiu pelo interesse na investigação dos riscos de lesões que ocorrem devido a execução incorreta de exercícios físicos, que aliado à outros fatores podem ocasionar as tendinopatias. Por essa razão, identificar a incidência de lesões tendíneas e analisar a prevalência destas nas academias de ginástica na cidade de Jataí - Goiás é o primeiro passo para a elaboração de formas preventivas e adequação de um diagnóstico preciso. A busca por esses dados somados aos estudos de trabalhos presentes na literatura é o que motiva a necessidade de adotar medidas de prevenção na realização de exercícios. Portanto, os objetivos do presente estudo foram identificar o perfil e verificar a prevalência de lesões osteomusculares de praticantes de exercício físico supervisionado em academia da cidade de Jataí-GO.

\section{MATERIAL E MÉTODO}

Delineamento e ética em pesquisa: 
Trata-se de um estudo pesquisa descritivo quantitativo transversal exploratório (GOMES, 2013). O estudo do tipo transversal ou de prevalência é usado para caracterizar a etiologia das doenças, sendo útil na avaliação da saúde populacional (BONITA et al, 2010).

O estudo foi aprovado pelo comitê de ética em pesquisa da Universidade Federal de Jataí sob o parecer $\mathrm{n}^{\circ}$ 59175816.0.0000.5083.

\section{Universo da Pesquisa e população alvo}

A pesquisa foi realizada em academias de ginástica da cidade de Jataí-GO. Inicialmente foi feito levantamento junto à Secretária de Esportes da prefeitura municipal para verificar a localização geográfica das academias na cidade. Depois foram selecionadas as academias que estavam cadastradas no Conselho Federal de Educação Física do estado de Goiás (CREF/14 GO-TO), destas foram selecionadas 10 instituições distribuídas em diferentes regiões da cidade. Foram adotados os seguintes critérios de elegibilidade para a seleção das academias de ginástica para realização da pesquisa: a) Oferecessem diversas modalidades de exercício físico (individuais e coletivas) com supervisão profissional;

b) Funcionassem em três períodos diários (manhã, tarde e noite);

c) Atendessem população de diversas faixas etárias e ambos os sexos.

Após o a seleção os proprietários das academias foram contatados, esclarecidos os procedimentos e, assim assinaram o termo de anuência para permanência do pesquisador no local e realização da coleta de dados.

Foram convidados a participar da pesquisa praticantes de atividades físicas supervisionadas de ambos os sexos acima de 18 anos de idade.

\section{Instrumento e coleta}

Foi elaborado um questionário semi-estruturado com questões objetivas e de múltipla escolha constituído com as seguintes dimensões:

a) Identificação, sexo e variáveis antropométricas (massa corporal e estatura), uso de tabaco ou fumo

b) Perfil e prática de atividade física supervisionada; tempo de prática, modalidade mais praticada, frequência semanal, prescrição e acompanhamento, realização de alongamento e aquecimento durante as sessões. 
c) Perfil de lesão ortopédica: tipo, local, classificação da dor e procedimento do praticante após lesão. Para avaliação do grau da dor foi utilizado a classificação proposta por (MELLION, 1997)

d) Grau I: Dor somente após a atividade;

e) Grau II: Dor com a atividade, que não restringe, mas pode afetar a performance;

f) Grau III: Dor com a atividade, que restringe e afeta de moderada a severamente a performance;

g) Grau IV: Dor com atividade e em repouso;

h) A coleta foi realizada em 10 academias em horários alternados de acordo com a disponibilidade do pesquisador. Os praticantes foram abordados de forma aleatória e convidados a participarem da pesquisa, uma vez, prontificados foi realizado a leitura dos procedimentos científicos e assinado o termo de livre consentimento esclarecidos pelos participantes. O questionário foi respondido pelo voluntário sem interferência do pesquisador. Os dados foram coletados e mantidos em sigilo.

i) Análise estatística

j) Depois de organizados e tabulados, os dados foram analisados pelo programa IBM SPSS Statistics versão 20. Após o teste de normalidade Kolmogorov-Smirnov, foi realizado o teste do Qui quadrado com o recurso da análise de V de Cramer e avaliação residual para avaliar as possíveis relações entre as variáveis. O nível de significância adotado foi $\mathrm{p}<0,05$.

\section{RESULTADOS E DISCUSSÃO}

Foram aplicados 200 questionários em 10 academias de ginásticas na cidade Jataí - GO. A escolha da amostra $(\mathrm{N}=200)$ embasou-se em outros estudos na área, pois os proprietários de academias previamente contatados optaram por não passar os dados sobre o número de alunos matriculados. O critério de escolha partiu da relevância do número de habitantes da cidade, com a população alvo de interesse (praticantes de musculação). Esse número se comparado às pesquisas que utilizaram $\mathrm{N}=50$ para uma população de 249.477 mil 


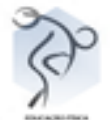

habitantes (WAGNER, 2010), 355.014 mil habitantes (SILVA, 2010), 10.539 mil habitantes (GOMES, 2013) e N=84 de um universo de 369.259 mil habitantes (REIS, 2015); mostra a significância relativa do público entrevistado, uma vez que, a cidade de Jataí possui 98.128 mil habitantes (IBGE, 2017).

A idade média dos participantes foi de $28,05 \pm 9,89$, variando 18 à 86 anos. O intervalo espaçado entre a idade mínima e a máxima é explicado por questões e justificativas inerentes a cada etapa da vida. Enquanto para os mais jovens a busca pelo ambiente de academia visa alcançar padrões de beleza impostos pela sociedade, os mais idosos buscam o envelhecimento saudável, com a prática musculação pelos seus benefícios físicos, emocionais e sociais (MEDEIROS et al., 2014, MASCARENHAS et. al., 2007; MARCELINO, 2003). A faixa etária é um fator de risco para ocorrência de lesões, a incidência das tendinopatias é mais predominante em pessoas acima dos 55 anos. Por isso o avanço progressivo da idade é diretamente proporcional ao aumento nas chances de ocorrerem lesões (PRADO, 2013). Pessoas idosas são mais suscetíveis a sofrerem danos pelas modificações na hidratação do colágeno, rigidez articular, diminuição da flexibilidade e da densidade óssea (LAURINO, 2008). Em contrapartida pessoas mais jovens podem se lesionar, não pela perda das características anatomofuncionais, mas pela sobrecarga de peso e séries mais extenuantes (HALL, 2000). Os dados obtidos no presente trabalho, reforçam os estudos encontrados na área, e mostram que 99,5\% dos entrevistados encontram-se na faixa etária até 55 anos, o que favorece a aptidão física com menores riscos de lesões.

Em relação ao sexo dos participantes da pesquisa, não há variância significativa, 105 eram homens e 95 mulheres. Não foram observadas significância quanto a esse quesito, sendo que a correlação entre lesões e sexo não foram observadas. Para alguns autores, o sexo pode ser visto como um fator intrínseco para o risco de lesões. As mulheres possuem articulações mais flexíveis, menor massa muscular e menor massa óssea, sendo mais vulneráveis a se lesionarem (PRADO, 2013). Entretanto, a anatomia masculina favorece o treino com demandas maiores de cargas para o aumento muscular desejado. Pela cultura em que estão inseridos, os homens almejam muito mais crescimento muscular do que as mulheres, que buscam um corpo mais definido. Assim, estão mais expostos aos possíveis 


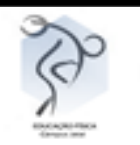

danos provocados pela musculação, principalmente nos grupos musculares da região superior do corpo (peitorais, braços, ombros e costas) (WAGNER, 2013).

Quanto ao tabagismo, é perceptível a discrepância entre os fumantes e não fumantes, 176 participantes responderam que não são e apenas 24 afirmaram ser tabagistas. $\mathrm{O}$ estilo de vida é um fator influente que facilita ou dificulta a ocorrência de lesões. O tabagismo em questão contribui para diminuição da capacidade pulmonar que resulta na menor oferta de oxigênio para os tendões e subsequente degeneração por hipóxia (GERE-MIA, 2011).

Em relação ao tempo de prática com exercícios físicos os entrevistados frequentavam a academia, 70 pessoas responderam que frequentavam a menos de seis meses, 41 marcaram a opção (outros) que corresponde a mais de 03 anos, 36 responderam de 06 meses a 01 ano, 33 de 1 a 2 anos e 20 de 2 a 3 anos. A correlação entre o tempo de musculação e a tendinite normalmente é caracterizada à longo prazo, pelo uso repetitivo ou excessivo dos tendões e por efeito acumulativo de micro traumas (ROSSETO, 2009).

A exposição constante, excessiva e prolongada em qualquer modalidade esportiva aumenta os riscos de ocorrerem lesões crônicas (AGUIAR et.al., 2010; ALMEIDA, 2003). Esses dados corroboram com os do presente trabalho em que 66\% dos entrevistados que afirmam ter tendinite) praticavam academia a mais de um ano. Em contrapartida, 96 entrevistados frequentavam a academia a menos de um ano, por isso acredita-se que a variável do dano progressivo não esteja interferindo nesses casos. No entanto, a falta de regularidade nas sessões de treinamento físico associado com cessão da prática regular promovem o destreina mento e desadaptações morfofuncionais que podem resultar em trauma súbito na estrutura tendínea (SANDMEIER; RENSTROM, 1997).

Frequência Semanal de Treinamento Físico Assim, nessa pesquisa foi relevante analisar as consequências do excesso e da falta de regularidade dos treinos diários dos participantes observados na questão 04. Nota-se que 74 pessoas responderam que frequentaram a academia de 4 a 5 vezes por semana, 70 de 5 a 6 vezes, 34 de 3 a 4 vezes, 18 de 2 a 3 vezes e apenas 04 afirmaram frequentar de 1 a 2 vezes por semana. O presente estudo demonstrou que a maioria das pessoas tem praticado musculação regularmente durante a maior parte da semana. A teoria dos benefícios da prática regular de atividades físicas aliados à uma orientação profissional correta, afirma que o exercício não danifica a estrutura tendínea, 


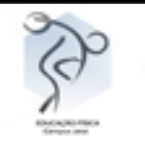

mas sim, estimula a síntese de novas fibras de colágeno tornando o tendão mais forte e resistente aos possíveis danos teciduais (QUINTANILLA, et al., 2012). Dos entrevistados que relataram ter tendinite, 20 descrevam que treinam de 4-6 vezes na semana. Sessões de treinamento extenuantes e com sobrecarga de carga e realizados com alto número de repetições e alta intensidade, estão relacionados a prejuízos na estrutura tendínea (CHEUNG; HUME; MAXWELL, 2003). As lesões adquiridas pela falta de descanso correto e excesso de treino ocorrem por estresse tecidual gerando micro traumas. A persistência da lesão associada com treinos intensos resulta em lesões graves, na qual o atleta poderá ficar incapacitado até mesmo para os exercícios diários (SILVA, 2010).

Um dos fatores que contribuem para a prevenção de lesões foi abordado na questão 05 concomitante com a questão 09 do questionário, que questiona sobre o aquecimento e alongamento respectivamente. Do total de entrevistados, 136 pessoas responderam que fazem aquecimento menos de trinta minutos, 41 afirmaram que não fazem, 15 até trinta minutos e 08 mais de trinta minutos. A respeito do alongamento, 55 responderam que fazem somente antes do exercício, outras 55 pessoas afirmaram que fazem antes e depois dos exercícios, 53 disseram que não fazem alongamento, 35 fazem apenas depois dos exercícios e 02 não responderam. Observa-se respectivamente que 79,5\% e 77,5\% das pessoas entrevistadas realizam algum tipo de aquecimento e alongamento. Esses dados refletem a realização de práticas adequadas de treinamento físico com maior eficiência energética e diminuição nos riscos de lesões. Isso ocorre porque o aquecimento é responsável por diversas atividades metabólicas que visa preparar o organismo para o treino de carga subsequente. Esses mecanismos permitem o aumento da temperatura muscular, redução da rigidez articular, aumento da capacidade circulatória pulmonar que resulta na maior oferta de oxigênio para os tecidos e para corrente sanguínea, além de ativar a atividade enzimática acelerando os processos metabólicos necessários à atividade física (JUNIOR et.al., 2014).

Dos entrevistados que relataram ter tendinite 23 (69\%) afirmam realizar aquecimento com tempo inferior a 30min. O aquecimento é a parte inicial de uma sessão de exercício físico da atividade física, sem essa preparação ou uma preparação inadequada (duração curta abaixo de 30 minutos) não haverá o aumento ideal da temperatura. $\mathrm{O}$ calor liberado pela contração das fibras musculares promove a liberação de $\mathrm{O} 2$ pela hemoglobina, 


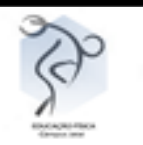

que aumenta o aporte sanguíneo para a atividade do músculo (ALEN-CAR; MATIAS, 2010). Sem esse mecanismo adequado de oxigenação, a hipóxia resultante do processo metabólico inadequado pode culminar em micro lesões tendíneas.

Em associação ao aquecimento, o alongamento contribui favoravelmente para o aumento da flexibilidade. Uma vez que, essa técnica, amplia a extensibilidade musculotendínea e do tecido conjuntivo muscular (ALMEIDA et.al., 2009). Além de promover a ação de "economia de energia", que ocorre quando o tecido tendíneo é colocado em tensão e a energia elástica armazenada é liberada posteriormente favorecendo o aumento da força muscular (WITVROUW et al., 2007). O alongamento realizado antes de iniciar qualquer atividade física proporciona que a musculatura alcance amplitude máxima de movimento. Além disso, prepara os tendões, de forma que suas camadas se lubrifiquem, e submetam-se a uma força de tração, para que estejam propícios para o exercício a seguir. No entanto deve ter duração de 30 a 45 segundos por músculo (AKAGI; TAKAHASHI, 2013). Os exercícios de alongamento realizados pós-treino, têm a intenção de causar micro lesões nas fibras tendíneas, na qual possibilita que elas sejam esticadas e regeneradas com um comprimento maior, com o passar do tempo. Nesse caso, as sessões devem ser mais de 60 segundos por músculo de maneira constante (KAY; BLAZEVICH, 2012). Porém, o alongamento pré-exercício que ultrapassa 60 segundos, diminui a força, velocidade e potência da performance esportiva, aumentando assim o risco de lesões (COSTA et. al., 2014). Das 33 pessoas que tiveram tendinite, 13 afirmaram que fazem alongamento antes do exercício. Estudos têm demonstrado que o alongamento pré-exercício não reduz as chances de ocorrer lesões, e pode predispor a lesão quando realizados de maneira errônea ou insuficiente (ALMEIDA, et. al., 2009).

Os entrevistados também foram questionados quanto elaboração e prescrição do programa de treinamento de exercício resistido proposto pelo professor na academia, 99 alunos afirmaram que sempre seguem as orientações, 73 não seguem porque, segundo eles, tem conhecimento para realizar seus treinamentos sem acompanhamento, 28 seguem às vezes. De acordo com esses dados, a maioria dos voluntários recorrem a orientação e prescrição do treinamento resistido por profissionais é bem instruída na academia e consequentemente sofrem menos prejuízos. O exercício resistido quando realizados e acompanhados, adequadamente, apresentam baixas taxas de lesões quando comparados com outros esportes 


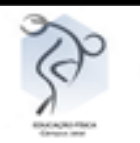

(SIMÃO, 2004), porém se o treinamento for mal elaborado sem princípios científicos e mal executado, podem acarretar em sérias complicações de efeito imediato ou à longo prazo (ROSSETO, 2009).

Nesse contexto, observa-se que 163 participantes responderam não ter apresentado lesão, o que representa $81,5 \%$, e 20 pessoas relataram lesão no músculo, representando $10 \%$ do todo. Ao passo que 07 dos investigados afirmaram ter sofrido na articulação, 06 pessoas tiveram lesões mas não souberam definir, 03 tiveram no tendão e 01 no osso. Como ilustrado no gráfico abaixo:

Gráfico 1: Lesões no sistema musculoesquelético relatadas por praticantes de academia de ginástica, Jataí, GO, 2017.

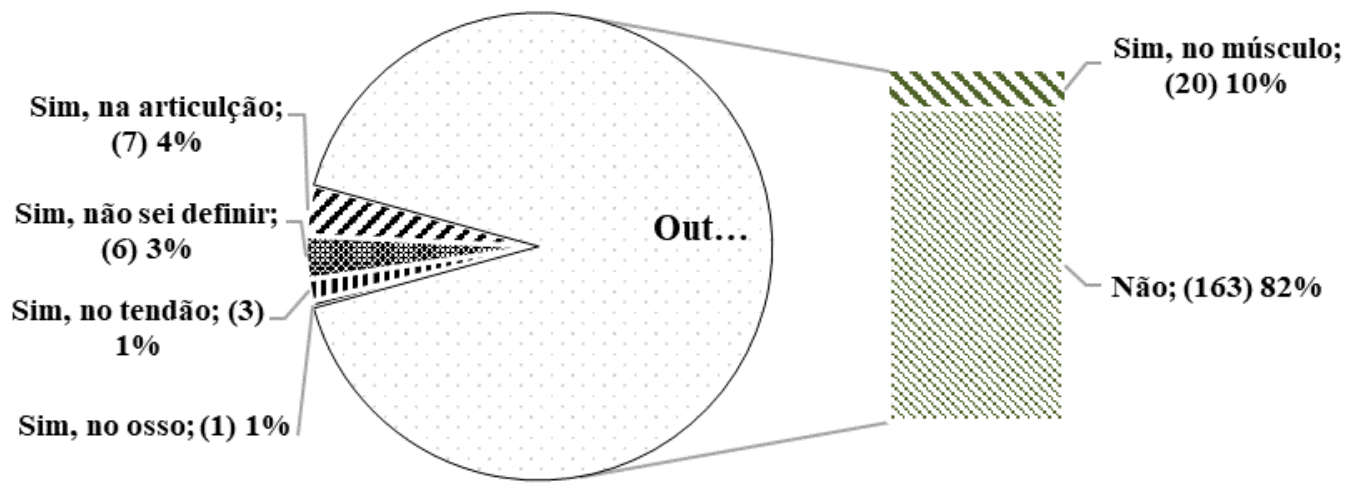

As lesões musculares foram as mais frequentes quando comparadas a outros locais de lesão, esses dados corroboram com os descritos por PRADO, 2013. Isso ocorre porque o sistema musculo esquelético é comumente usado para todo o fim esportivo, mas se essa atividade for realizada com excesso de peso, esforços repetitivos e sem uma orientação adequada, pode trazer prejuízos maiores a saúde (REIS, 2015). As lesões musculares são induzidas pelo exercício físico excessivo, com desenvolvimento de fadiga muscular por depleção nos estoques de glicogênio (NOAKES, 2000; TEE, BOSCH, LAMBERT, 2007). As pessoas acometidas, geralmente continuam suas atividades logo após a lesão (SILVA, 2010). 


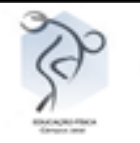

Muitos acreditam que a dor muscular é parte do processo de hipertrofia, e progridem em seu treinamento com aumento de carga ou intensidade (LOPES, 2009). Assim podem ocorrer lesões agudas ou crônicas. Entretanto, o que é mais perceptível no gráfico, são as pessoas que afirmaram não ter tido lesão, com o maior percentual de 81,5\%. Esses dados mostram que os participantes da pesquisa têm sido bem orientados nas academias, o que diminui os riscos de se lesionarem.

As lesões no esporte podem estar associadas a pessoas que além da musculação praticam qualquer outro tipo de exercício físico. A utilização excessiva sem a recuperação apropriada pode ocasionar deterioração tendínea pela sobrecarga de peso/atividades, agravando o quadro da doença ou gerando ruptura do mesmo (WAGNER, 2013). Por isso a prática de outras atividades físicas associadas ou não à a prática de exercício resistido foi estudada na presente pesquisa. Nota-se que 98 participantes disseram não praticar outros exercícios, 50 afirmaram realizar exercícios funcionais, 25 marcaram a questão (outros), 17 realizaram aulas coletivas e 10 praticavam lutas. Dos entrevistados, $49 \%$ só fazem musculação, o que evita a sobrecarga e favorece a preservação a musculatura esquelética e tendínea. Além disso 25\% relataram realizar exercícios funcionais com acessórios em aulas coletivas. Esse tipo de treinamento baseia-se em uma prescrição segura de exercícios que permitem que o sistema musculoesquelético e seus sistemas interdependentes sejam estimulados e melhorados. Assim, à melhora do condicionamento físico; os riscos de lesões diminuem e o retorno às atividades de indivíduos lesionados é mais rápido e seguro (RIBAS, 2016). O treinamento com pesos, quando realizados de maneira correta, pode ser profilático e terapêutico para muitas enfermidades esportivas (SILVA, 2010). Esses dados confirmam os demais já descritos nessa pesquisa, demonstrando que os fatores de risco são evitados pelos praticantes de musculação em academias de ginástica em Jatai, Goiás.

Um total de 142 pessoas respondeu não ter tido tendinite, representando percentual de $71 \%$. Em contrapartida, 33 afirmaram que tiveram tendinite e 25 participantes não souberam responder (grafico 2).

Gráfico 2: Número e percentual das respostas sobre tendinite relatadas por praticantes de academia de ginástica, Jataí, GO, 2017. 
Você já teve tendinite?

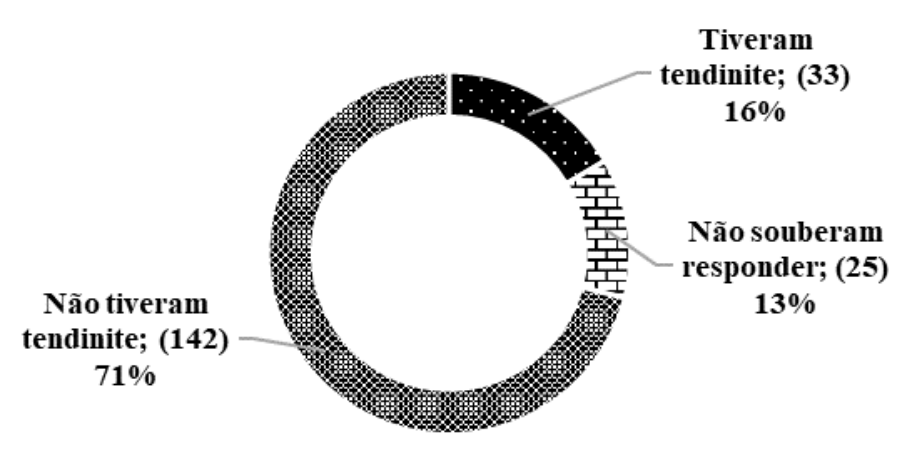

Não souberam responder $13 \%$ do total de entrevistados. Isso demonstra a falta de conhecimento das pessoas para com a doença. A tendinite tem o exames fisicos realizados por um médico (HERTLING; KESSLER, 2009). A problemática é que sem esse conhecimento, as pessoas tendem a continuar com suas atividades, mesmo com dor, e assim agravam o quadro pelo uso repetitivo da estrutura tendínea (SILVA, 2010). Diante disso, é necessário que as pessoas estejam esclarecidas a respeito dessa doença para que possam observar os sinais e procurar o devido tratamento.

Uma minoria tem sofrido com essa doença, mas devem ser considerados. Esses dados seguem no segundo gráfico:

Gráfico 3: Articulações acometidas com tendinites em praticantes de exercício físico (exercício resistido) 


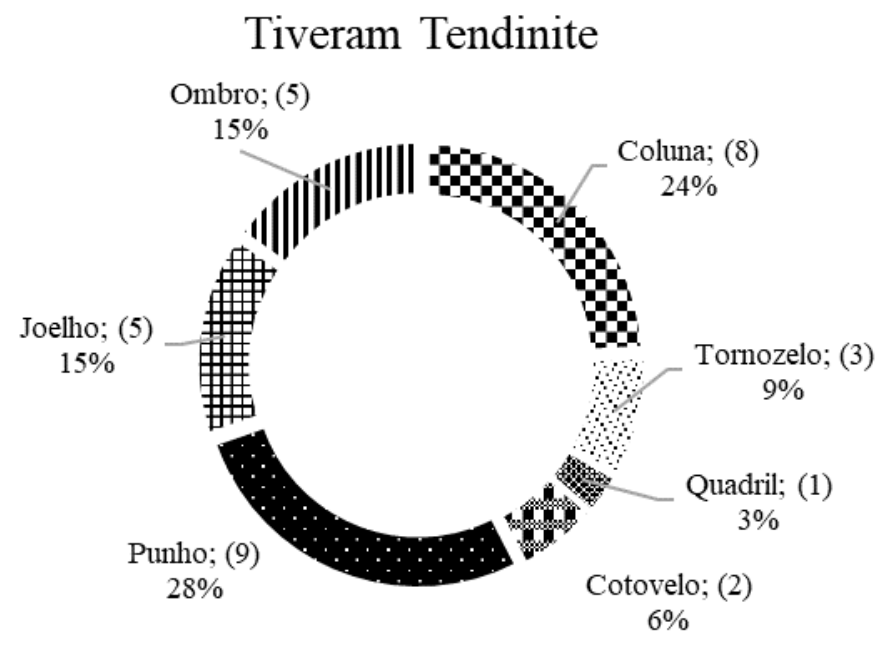

A tendinite ocorre devido a execução excessiva ou incorreta dos tendões nos exercícios de musculação (ROSSETTO, 2009). O gráfico 3 aponta que $28 \%$ das tendinites se concentra no punho, seguido da coluna (24\%) e ombro (15\%). Esses dados correlacionam-se com os descritos na literatura e podem não estar somente associados a prática de musculação, mas também a lesões anteriores. Dedos, mãos, punho, região cervical e região escapular são os mais utilizados na prática diária de trabalho manual, e consequentemente alvos frequentes para a ocorrência de tendinopatias (CIARLINI et. Al., 2005). Praticantes de modalidades esportivas podem apresentar lesões prévias não diagnosticadas ou sem manifestação clínica referente ao cotidiano de trabalho. Profissões que exigem esforço físico intenso, movimentos repetitivos e outros fatores de riscos (LER, DORT) quando associadas à exercícios de musculação, aumentam os riscos de lesões com maior intensidade e cronicidade (LELIS et.al., 2012; MAGAS et.al., 2012). Dentre os entrevistados que relataram ter tendinite, 14 pessoas não realizavam outra atividade além da musculação, o que reforça que essas pessoas já tenham a doença em sua forma subclínica.

A dor é o principal sintoma na presença de lesão, ela reflete diretamente o grau de severidade da doença em questão, sendo proporcional aos danos pelo uso excessivo das estruturas (CARDOSO, 2011). Isso implica os dados da questão 08 do questionário que aborda a classificação da dor, na qual 159 pessoas não responderam por não sentirem dor ou 


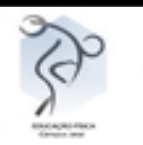

terem algum tipo de lesão. Porém, 14 afirmaram que sentem dor somente após a atividade, 14 sentem dor durante a atividade, que não restringe, mas afeta a performance, 12 sentem dor com atividade e em repouso, e 01 pessoa marcou que sente dor com atividade, que restringe e afeta de moderada a severamente a performance. A dor somente após a atividade física, relatada por 7,0\% dos praticantes pode estar relacionada aos mecanismos da dor muscular tardia. Esses episódios são caracterizados por uma gama de fatores etiológicos diferentes, que vão desde fadiga por ausência de preparo físico (estresse metabólico) até danos na estrutura muscular provocado por forças mecânicas elevadas (estresse mecânico) (TRICOLI, 2001).

Os dados do estudo indicam que a dor relatada está relacionada a adaptações fisiológicas ao treinamento físico, uma vez que 34\% dos entrevistados descreveram frequentar a academia a menos de seis meses. O período de adaptação a pesos e treino geralmente é acompanhado por pequenas dores musculares, deve-se, entretanto, atentar-se a essas dores e observar. Dependendo da intensidade dos exercícios de carga, por efeito do trauma acumulativo, pode haver o enfraquecimento das fibras colágenas e modificações na sua estrutura molecular, o que pré-dispõe o surgimento das tendinopatias (MAGAS et.al., 2012). Os demais entrevistados (27 pessoas) que relataram ter dores descrevem que estas os acompanham em intensidades variadas durante a realização dos exercícios, e 96,3\% relataram já terem tido tendinite. A presença de dor na realização de atividades físicas está associada às lesões degenerativas com lacerações microscópicas e fadiga tecidual (HERTLING; KESSLER,2009). A insistência em continuar a realizando de exercícios físicos, mesmo sentindo dor, pode trazer prejuízos severos e até mesmo irreversíveis para a pessoa em questão (HAYEM, 2001).

A análise das pessoas que não responderam sentir dor, correspondente a 79,5\%, e permite compreender que o público alvo da pesquisa, em sua maioria, não apresentaram sintomas clínicos da doença. Nota-se que a quantidade de pessoas que disseram não ter lesão devido a prática da academia juntamente com não ter tido tendinite, é diretamente proporcional ao número de pessoas que fazem aquecimento e alongamento corretos antes de realizarem atividades físicas. Esses dados corroboram com os dados da questão 6 e 7 do questionário, em que $81,5 \%$ apresentaram não ter tido alguma lesão devido a prática da academia e $71 \%$ não tiveram tendinite 
Observam-se nos dados pertinentes à questão 10, que indaga sobre a atitude após a lesão, que 12 dos entrevistados não procuraram um médico e continuaram a realizar as atividades, 13 procuraram um médico, 04 não procuraram um médico e deixaram de fazer apenas algumas atividades e 04 não procuraram um médico e deixaram de realizar todas as suas atividades. Essas respostas são proporcionais às respostas anteriores sobre a percepção das pessoas quanto às lesões e dor. Somente 39\% procuram ajuda médica, enquanto $60 \%$ ignoraram a busca pelo profissional. Para que haja o relato da lesão, é imprescindível que o indivíduo acometido tenha a percepção da ocorrência da mesma. A busca por um médico é o início do diagnóstico, que por muitas vezes é difícil e impreciso, sendo necessária cautela na interpretação clínica, aliada a anamnese detalhada e exames físicos (SIENA \& HELFENSTEIN, 2009).

Os resultados evidenciaram a percepção das pessoas quanto a lesões relacionadas às atividade realizadas nas academias, ou dores resultantes da prática de musculação. A análise da pesquisa reflete que os alunos das academias exploradas estão mais instruídos ou estão seguindo mais frequentemente a orientação do professor, e cada um respeitando mais seus próprios limites de sobrecarga (GOMES, 2013). Por ser um estudo transversal exploratório de base individual, os resultados aqui encontrados podem servir como suporte para estudos posteriores que desenvolvam estratégias de intervenção e prevenção de tendinopatias e lesões em praticantes de musculação nas academias de ginástica.

\section{CONCLUSÃO}

De acordo com os resultados obtidos na pesquisa, observou-se que a maioria da população estudada têm alcançado benefícios e ausência de dor/tendinopatias com prática de exercícios físicos quando supervisionados de forma adequada. Porém, quando realizados de maneira errônea e com excesso de sobrecarga resultaram em lesões como a tendinite.

A partir disso, enfatiza-se a importância do conhecimento e detecção de lesões ortopédicas pelo profissional de Educação Física para prescrição adequada e orientação em caso de detecção de anormalidades osteomusculares. 


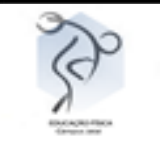

\section{REFERÊNCIAS}

AKAGI, R.; H. TAKAHASHI. Acute Effect of Static Stretching on Hardness of the Gastrocnemius Muscle. Med. Sci. Sports Exerc, Vol. 45, No. 7, pp. 1348-1354, 2013.

ALBUQERQUE, C. V.; MASCHIO, J. P.; GRUBER, C. R.; SOUZA, R. M.; HERNANDEZ, S. Efeito agudo de diferentes formas de aquecimento sobre a força muscular. Fisioter. Mov., Curitiba, v. 24, n. 2, p. 221-229 abr./jun, 2011.

ALENCAR, T. A. M.; MATIAS, K. F. S. Princípios fisiológicos do aquecimento e alongamento muscular na atividade esportiva. Rev Bras Med Esporte vol.16, n.3, Niterói, May/June, 2010.

ALMEIDA, P. H. F.; BARANDALIZE, D.; RIBAS, D. I. R.; GALLON, D.; MACEDO, A. C. B.; GOMES, A. R. S. Alongamento muscular: suas implicações na performance e na prevenção de lesões. Fisioter. Mov., Curitiba, v. 22, n. 3, p. 335-343, jul./set. 2009.

BERTOLINI, G. R. F.; SILVA, T. S.; CIENA, A. P.; ARTIFON, E. L. Comparação do ultrassom pulsado e contínuo no reparo tendíneo de ratos. Laboratório de Estudo das Lesões e Recursos Fisioterapêuticos da Universidade Estadual do Oeste do Paraná (UNIOESTE). Cascavel, 2012.

CIARLINI, I. A.; MONTEIRO, P. P.; BRAGA, R. O. M.; MOURA, D. S. Lesões por esforços repetitivos em fisioterapeutas. Universidade de Fortaleza, Fortaleza-Ceará, Brasil. Revista Brasileira em Promoção da Saúde, 2005, vol. 18, núm. 1, pp. 11-16. Disponível em: http://www.redalyc.org/articulo.oa?id=40818104. Acesso em: 04/07/2016.

CIVINSK, C.; MONTIBELLER, A.; BRAZ, A. L. O. A importância do exercício físico no envelhecimento. Revista da Unifebe, 2011, p. 163-175.

CORTES, A. A.; MONTENEGRO, A.; AGRA, A. C.; ERNESTO, C.; ANDRADE JR, M. S. A influência do treinamento de força na flexibilidade. Rev Digit Vida Saúde. v.1, n.2, p.1-6, 2002.

COSTA, P. B., T. J. HERDA, A. A. HERDA, and J. T. CRAMER. Effects of Dynamic Stretching on Strength, Muscle Imbalance, and Muscle Activation. Med. Sci. Sports Exerc., Vol. 46, No. 3, pp. 586-593, 2014.

DALLA NORA, D.; PETTER, G. N.; SANTOS, T. S.; BRAZ, M. M.; LEMOS, J. C. Fatores relacionados ao encurtamento dos isquiotibiais: um estudo bibliográfico. In: $3^{\circ}$ Fórum de Fisioterapia, 2012, SANTA MARIA. EDIÇÃO ATUAL - Anais Fórum de Fisioterapia Unifra. vol 1. Santa Maria: 2012. v. 1. Disponível em: http://www.unifra.br/eventos/forumfisio/Trabalhos/5079.pdf. Acesso em: 08/07/2016. 


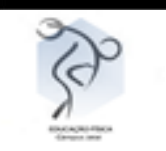

GEREMIA, J. M. Ruptura total do tendão de aquiles: propriedades mecânicas tendíneas em indivíduos submetidos a diferentes protocolos de reabilitação. Porto Alegre, 2011.

GOMEZ, V. S. Análise dos principais tipos de lesões em praticantes de musculação na cidade de Cachoeira Alta - GO, Goiânia: EdUFG, 2013.

HALL, S. J. Biomecânica Básica. Rio de Janeiro: Guanabara Koogan, 2000.

IRIART, J. A. B.; CHAVES, J. C.; ORLEANS, R. G. Culto ao corpo e uso de anabolizantes entre praticantes de musculação. Cad. Saúde Pública, 25(4):773-782, Rio de Janeiro, 2009.

JORGE, R. T. et.al. Treinamento resistido progressivo nas doenças musculoesqueléticas crônicas. Rev. Bras. Reumatol. vol.49 no.6 São Paulo Nov./Dec. 2009.

KAY, A. D., and A. J. BLAZEVICH. Effect of Acute Static Stretch on Maximal Muscle Performance: A Systematic Review. Med. Sci. Sports Exerc., Vol. 44, No. 1, pp. 154-164, 2012.

LAURINO, C. F. S. Atualização em ortopedia e traumatologia. 1. ed. São Paulo: Office Editora, v. 1. 48p, 2012.

LOPES, A. S. Avaliação do efeito de um programa de treino de musculação na dor e na funcionalidade da região lombar. Porto, 2009.

LELIS, C. M.; BATTAUS, M. R. B.; FREITAS, F. C. T.; ROCHA, F. L. R.; MARZIALE, M. H. P.; ROBAZZI, M. L. C. C. Distúrbios osteomusculares relacionados ao trabalho em profissionais de enfermagem: revisão integrativa da literatura, Universidade de São Paulo. Escola de enfermagem de Ribeirão Preto; 2012.

MACÊDO, P. R. S.; COSTA, R. C. S.; SOUZA, C. G.; LEITE, E. C. F. Intervenções fisioterapêuticas na tendinopatia do ombro: uma revisão de literatura. In: $4^{\circ}$ Congresso Internacional de Envelhecimento Humano, 2015, Campina Grande. Anais CIEH (2015) Vol. 2, N.1. Campina Grande: Centro Multidisciplinar de Estudos e Pesquisas (CEMEP), Universidade Estadual da Paraíba, 2015.

MAGAS, V.; NEVES, E. B.; MOURA, M. A. M. DE; NOHAMA, P. Avaliação da aplicação da termografia no diagnóstigo de tendinite de punho por ler / dort.. Anais do XXIII Congresso Brasileiro de Engenharia Biomédica. Recife: Altech. Porto de Galinhas. v. 1. p. 583-586, 2012.

MARTINS, A. P. C.; GOMES, A. G. S.; PEREIRA, G. S.; FERNANDES, L. S.; BADARÓ, R. R.; ALVES, T. C. F. Incidência de lesões em praticantes de musculação nas academias de ginástica da cidade de Guanambi - BA. 


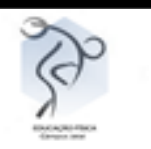

MEDEIROS, J. J. de; BRITO, M. V. G.; PERRACINI, M. R.; ARAÚJO, F. B. de; SANTOS, A. D. Aplicabilidade de hidroginástica e musculação em pessoas idosas da comunidade. Revista Interfaces: Saúde, Humanas e Tecnologia, v. 2, n. 6, ano 2, 2014.

MEDONÇA, L. D. M.; MACEDO, L. G.; FONSECA, S. T.; SILVA, A. A. Comparação do alinhamento anatômico de membros inferiores entre indivíduos saudáveis e indivíduos com tendinose patelar. Rev. bras. fisioter. Vol. 9, No. 1 (2005), 101-107.

MONTEIRO, A; CARNEIRO. T; O que é Treinamento Funcional? [s.1.] 20 de abril de 2010. Disponível em http://www.arturmonteiro.com.br/2010/04/o-que-etreinamentofuncional/; Acesso em: 05 out. 2016.

OLIVEIRA, R. R. Avaliação das propriedades mecânicas do tendão do calcâneo de ratos diabéticos submetidos ao treinamento físico aeróbico moderado. Recife: EdUFP, 2011.

PRADO, R. O. Lesões em esportes de contato no curso de educação física da Universidade Federal de Santa Catarina. Santa Catarina: EdUFSC, 2013.

REIS, E. M. Índice de lesões em academias de musculação de porto velho, Portoo Velho: EdUFR, 2015.

RIBAS, L. F. R. A. Treinamento funcional e sua importância na reabilitação. Manaus, 2016.

RIBEIRO, P. G. A. A efectividade do exercício excêntrico no tratamento da tendinopatia do tendão rotuliano - que particularidades estão associadas a melhores resultados? Porto: EdUFP, 2013.

ROLLA, A. L.; ZIBAOUI, N.; SAMPAIO, R. F.; VIANA, S. O. Análise da percepção de lesões em academias de ginástica de Belo Horizonte: um estudo exploratório. Revista Brasileira de Ciência e Movimento. v. 12, n. 2, p.7-12, 2004.

ROSSETTO, N. P. A viscoelasticidade no alongamento de tendões. Campinas: EdUEC, 2009.

SANTANA, N. L.; CAMPOS, L. A. S. Lesões na prática da musculação: aspectos fisiológicos e psicológicos analisados em academias de patos de minas - mg. Coleção Pesquisa em Educação Física - Vol.7, no 3 - 2008.

SIENA, C; HELFENSTEIN, M. Equívocos diagnósticos envolvendo as tendinites: impacto médico, social, jurídico e econômico, Rev Bras Reumatol 2009; 49(6):712-25. 


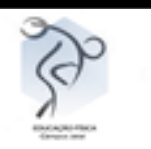

SILVA, A. V. A. Incidência de lesões em praticantes de musculação em ambiente de academia. Campina Grande: EdUEPB, 2010.

SILVA, L. P. A.; OLIVEIRA, M. F. M.; CAPUTO, F. Métodos de recuperação pósexercício. Florianópolis, v. 24, n. 3, 2013, p. 489-508.

SILVA, J. M. N.; CARVALHO, J. P.; MOURA JR, M. J. Estudo morfométrico da terapia led de baixa potência em tendinite de ratos. Fisioterapia e Pesquisa, São Paulo, v.18, n.4, out/dez. 2011, p. 365-70.

SILVARES. P. R. A. Gênero específico na medição vivo das propriedades estruturais e mecânicas do tendão patelar humano. Botucatu: EdUNESP, 2007.

SIMÃO, R.; POLITO, M.; MONTEIRO, W. Efeitos de diferentes intervalos de recuperação em um programa de treinamento de força para indivíduos treinados. Rev Bras Med Esporte - Vol. 14, No 4 - Jul/Ago, 2008.

TAHARA, A. K.; SCHWARTZ, G. M.; SILVA, K. A. Aderência e manutenção da prática de exercícios em academias. Laboratório de Psicologia do Esporte e do Exercício da Universidade do estado de Santa Catarina - UDESC, Florianópolis, SC. R. bras. Ci e Mov.; 11(4): 7-12, 2003.

TRICOLI, Valmor. Mecanismos envolvidos na etiologia da dor muscular tardia. Revista Brasileira de Ciência e Movimento, v. 9, n. 2, p. 39-44, 2008.

VIEIRA, F. F.; FERREIRA, L. A. B.; PEREIRA, W. M.; ROSSI, L. P. Aspectos histopatológicos nas tendinopatias. In: XI Encontro Latino Americano de Iniciação Científica e VII Encontro Latino Americano de Pós-Graduação, 2016, Guarapuava. Resumos. Guarapava: Universidade do Vale do Paraíba, 2016. p. 1100-1103.

WAGNER, E. Estudo de lesões musculares e articulares em praticantes de musculação de uma academia do município de Florianópolis-SC, Florianópolis: EdUFSC, 2013. 